\title{
Heparan Sulfate Induces Necroptosis in Murine Cardiomyocytes: A Medical-In silico Approach Combining In vitro Experiments and Machine Learning
}

\author{
Elisabeth Zechendorf ${ }^{1}$, Phillip Vaßen ${ }^{2}$, Jieyi Zhang ${ }^{2}$, Ahmed Hallawa ${ }^{3}$, \\ Antons Martincuks ${ }^{4}$, Oliver Krenkel ${ }^{1,5}$, Gerhard Müller-Newen ${ }^{4}$, Tobias Schuerholz ${ }^{6}$, \\ Tim-Philipp Simon ${ }^{1}$, Gernot Marx ${ }^{1}$, Gerd Ascheid ${ }^{3}$, Anke Schmeink ${ }^{2 *}$, Guido Dartmann ${ }^{7 *}$, \\ Christoph Thiemermann ${ }^{8^{\dagger}}$ and Lukas Martin ${ }^{1,8^{\dagger} *}$

\begin{abstract}
${ }^{1}$ Department of Intensive Care and Intermediate Care, University Hospital RWTH Aachen, Aachen, Germany, ${ }^{2}$ Research Area Information Theory and Systematic Design of Communication Systems, RWTH Aachen University, Aachen, Germany, ${ }^{3}$ Chair for Integrated Signal Processing Systems, RWTH Aachen University, Aachen, Germany, ${ }^{4}$ Institute of Biochemistry and Molecular Biology, RWTH Aachen University, Aachen, Germany, ${ }^{5}$ Department of Medicine III, University Hospital RWTH Aachen, Aachen, Germany, ${ }^{6}$ Department of Anesthesia and Intensive Care, University Hospital Rostock, Rostock, Germany, ${ }^{7}$ Research Area Distributed Systems, Trier University of Applied Sciences, Trier, Germany, ${ }^{8}$ William Harvey Research Institute, Queen Mary University London, London, United Kingdom
\end{abstract}

Life-threatening cardiomyopathy is a severe, but common, complication associated with severe trauma or sepsis. Several signaling pathways involved in apoptosis and necroptosis are linked to trauma- or sepsis-associated cardiomyopathy. However, the underling causative factors are still debatable. Heparan sulfate (HS) fragments belong to the class of danger/damage-associated molecular patterns liberated from endothelial-bound proteoglycans by heparanase during tissue injury associated with trauma or sepsis. We hypothesized that HS induces apoptosis or necroptosis in murine cardiomyocytes. By using a novel Medical-In silico approach that combines conventional cell culture experiments with machine learning algorithms, we aimed to reduce a significant part of the expensive and time-consuming cell culture experiments and data generation by using computational intelligence (refinement and replacement). Cardiomyocytes exposed to HS showed an activation of the intrinsic apoptosis signal pathway via cytochrome $\mathrm{C}$ and the activation of caspase 3 (both $p<0.001$ ). Notably, the exposure of HS resulted in the induction of necroptosis by tumor necrosis factor $\alpha$ and receptor interaction protein $3(p<0.05 ; p<0.01)$ and, hence, an increased level of necrotic cardiomyocytes. In conclusion, using this novel Medical-In silico approach, our data suggest (i) that HS induces necroptosis in cardiomyocytes by phosphorylation (activation) of receptorinteracting protein 3, (ii) that HS is a therapeutic target in trauma- or sepsis-associated cardiomyopathy, and (iii) indicate that this proof-of-concept is a first step toward simulating the extent of activated components in the pro-apoptotic pathway induced by HS with only a small data set gained from the in vitro experiments by using machine learning algorithms.

Keywords: septic cardiomyopathy, necroptosis, apoptosis, Petri nets, modeling, optimization, small data 


\section{INTRODUCTION}

Severe injuries and systemic infection are the leading causes of death in intensive care units worldwide (1). Post-injury organ failure is defined as a life-threatening condition caused by a dysregulated host response to trauma or infection resulting in dysfunction and ultimately failure of many organs. The heart is one of the most frequently affected organs in the multiple organ failure syndrome associated with sepsis and trauma $(2,3)$. Several studies indicate that cardiac apoptosis, necrosis, or necroptosis play a pivotal pathophysiological role in cardiomyopathy associated with trauma or sepsis (4-7). Apoptosis describes a programmed, cysteinyl aspartate-specific protease (caspase)-dependent form of cell death, whereas necroptosis is a programmed caspase-independent form of necrosis. While the activation of caspase 3 leads to apoptosis induction, the activation of mixed lineage kinase domainlike (MLKL) and receptor-interacting protein (RIP) 3 results in necroptosis $(8,9)$. RIP3 and the pseudokinase MLKL form a necrosome to induce necroptosis. The underlying mechanisms and causative factors that induce cardiac apoptosis or necroptosis in trauma or sepsis, however, are largely unknown.

Tissue injury after trauma or infection results in an increased expression of pro-inflammatory cytokines, such as tumor necrosis factor alpha (TNF- $\alpha$ ) and interleukin (IL)-6, both of which play a central role in the process of trauma and sepsis-associated cardiac dysfunction (10). Cytokines, such as IL-6, liberate and activate the endo-b-D-glucuronidase heparanase, a sheddase that specifically cleaves heparan sulfate (HS) fragments. HSs are highly sulfated glycosaminoglycans covalently attached to a core protein that is localized on the plasma membrane of endothelial cells (11). Once shed by heparanase, circulating HS fragments belong to the heterogeneous group of "danger/damageassociated molecular patterns (DAMPs)" (Figure 1). Indeed, patients with septic shock show elevated serum levels of HS fragments (12). Similar to molecules released by pathogens (pathogen-associated molecular patterns), HS interacts with pattern recognition receptors (e.g., toll-like receptors) on several cell types [including cardiomyocytes (12)], which results in an
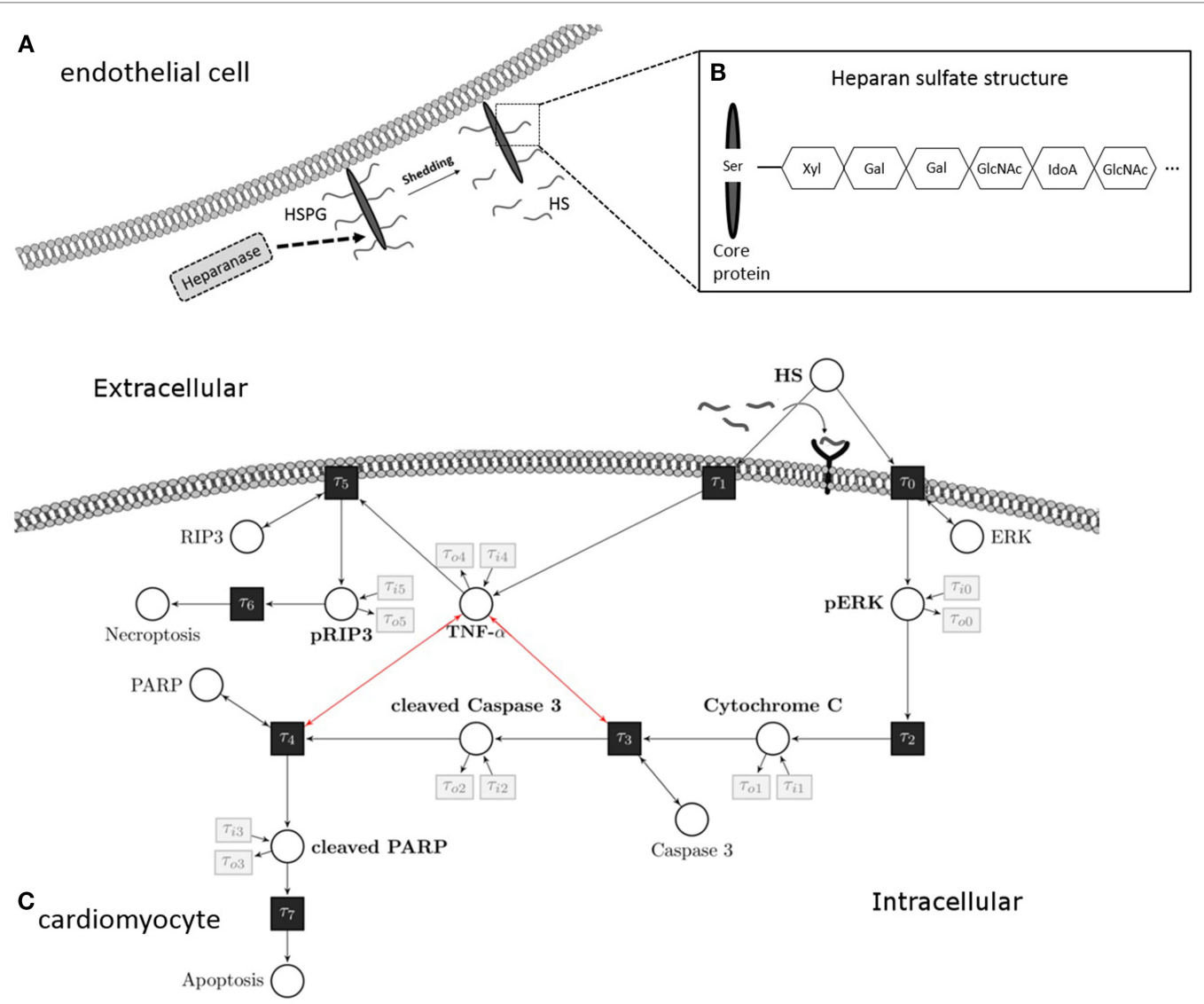

FIGURE 1 | Heparan sulfate induces an apoptosis/necroptosis signal pathway. (A) HS fragments are cleaved by heparanase from a HS proteoglycan, which is localized on the plasma membrane of endothelial cells. (B) Structure of HS proteoglycan. (C) HS interacts with a pattern recognition receptor (i.e., toll-like receptor 4) localized on the cell surface of cardiomyocytes and activates a pro-apoptotic intrinsic pathway. The signaling cascade involves phosphorylation of ERK $1 / 2$ resulting in the release of mitochondrial cytochrome $\mathrm{C}$, which leads to cleavage and activation of caspase 3 . In the next step of this pathway, PARP is cleaved and deactivated by activated caspase 3 . Induction of TNF- $\alpha$ caused by HS inhibits the pro-apoptotic pathway and induces phosphorylation of RIP3 and necroptosis. These apoptosis and necroptosis signal pathways represent our Petri net model. White circles are places, and black rectangles are transitions. Unidirectional arcs indicate directed flows. Bidirectional arcs indicate read arcs, which influence transitions, but do not consume tokens. Modified from Martin et al., Sarrazin et al., and Maeda (13-15). HS, heparan sulfate; PARP, poly-(ADP-ribose) polymerase; ERK, extracellular signal-regulated kinase; RIP, receptor-interacting protein; TNF- $\alpha$, tumor necrosis factor alpha; Ser, serine; Xyl, xylose; Gal, galactose; GlcNAc, N-acetylgalactosamine; IdoA, iduronic acid. 
TABLE 1 | Ordinary differential equations (ODEs) of the proposed Petri net model.

\begin{tabular}{lll}
\hline Label & Place & ODE \\
\hline Heparan sulfate & $p_{0}$ & $\dot{y}\left(p_{0}, t\right)=\frac{\partial H S(t)}{\partial t}$ \\
ph-ERK 1/2 & $p_{1}$ & $\dot{y}\left(p_{1}\right)=x_{11} x_{2}+x_{10}\left(x_{1} y\left(p_{0}\right)^{x_{9}}\right)-x_{11}\left(x_{2} y\left(p_{1}\right)^{\left.x_{11}\right)}\right.$ \\
Cytochrome C & $p_{2}$ & $\dot{y}\left(p_{2}\right)=\left(\frac{x_{13} x_{3} x_{26}}{x_{25} x_{26}+x_{25}+x_{26}}-x_{12} x_{2}\right)+x_{12}\left(x_{2} y\left(p_{1}\right)^{x_{11}}\right)-x_{3} y\left(p_{2}\right)^{x_{13}} \frac{x_{13} y\left(p_{2}\right)}{y\left(p_{2}\right)+x_{25}\left(1+y\left(p_{8}\right) / x_{26}\right)}$ \\
Cleaved caspase 3 & $p_{3}$ & $\dot{y}\left(p_{3}\right)=\frac{x_{15} x_{4} x_{28}}{x_{28} x_{27}+x_{28}+x_{27}}-\frac{x_{14} x_{3} x_{26}}{\left.x_{26} x_{25}+x_{26}+x_{25}\right)}+x_{3} y\left(p_{2}\right)^{x_{13}} \frac{x_{14} y\left(p_{2}\right)}{y\left(p_{2}\right)+x_{25}\left(1+y\left(p_{8}\right) / x_{26}\right)}-x_{4} y\left(p_{3}\right)^{x_{15}} \frac{x_{15} y\left(p_{3}\right)}{y\left(p_{3}\right)+x_{27}\left(1+y\left(p_{8}\right) / x_{28}\right)}$ \\
Cleaved PARP & $p_{4}$ & $\dot{y}\left(p_{4}\right)=\left(x_{17} x_{5}-\frac{x_{16} x_{4} x_{28}}{x_{28} x_{27}+x_{28}+x_{27}}\right)+x_{16} x_{4} y\left(p_{3}\right)^{x_{15}} \frac{y}{y\left(p_{3}\right)+x_{27}\left(1+y\left(p_{8}\right) / x_{28}\right)}-x_{17}\left(x_{5} y\left(p_{4}\right)^{\left.x_{17}\right)}\right.$ \\
ERK1/2 & $p_{5}$ & $\dot{y}\left(p_{5}\right)=0$ \\
Caspase 3 & $p_{6}$ & $\dot{y}\left(p_{6}\right)=0$ \\
PARP & $p_{7}$ & $\dot{y}\left(p_{7}\right)=0$ \\
TNF- $\alpha$ & $p_{8}$ & $\dot{y}\left(p_{8}\right)=x_{18} x_{7}+x_{20}\left(x_{6} y\left(p_{0}\right)^{x_{9}}\right)-x_{18}\left(x_{7} y\left(p_{8}\right)^{x_{18}}\right)$ \\
pRIP3 & $p_{9}$ & $\dot{y}\left(p_{9}\right)=x_{21} x_{8}-x_{19} x_{7}+x_{19}\left(x_{7} y\left(p_{8}\right)^{x_{18}}\right)-x_{21}\left(x_{8} y\left(p_{9}\right)^{\left.x_{21}\right)}\right.$ \\
RIP3 & $p_{10}$ & $\dot{y}\left(p_{10}\right)=0$ \\
\hline
\end{tabular}

They are constructed according to the given definition in Figure 8 for general mass action kinetics. Two special inhibiting transitions are inspired by Michaelis-Menten inhibition (37). These are connected by red bidirectional arcs to TNF- $\alpha$ within the Petri net (Figure 1). $x_{i}$ 's are unknown, hence arbitrary selectable, parameters which will be used for optimization. Optimized parameters and constant terms $c_{1}$ to $c_{8}$, which are added to establish an equilibrium initial state, are given in the online supplementary. HS, heparan sulfate; PARP, poly-(ADP-ribose) polymerase; ERK, extracellular signal-regulated kinase; RIP, receptor-interacting protein; TNF- $\alpha$, tumor necrosis factor alpha.

ALGORITHM 1 | Optimization Routine.

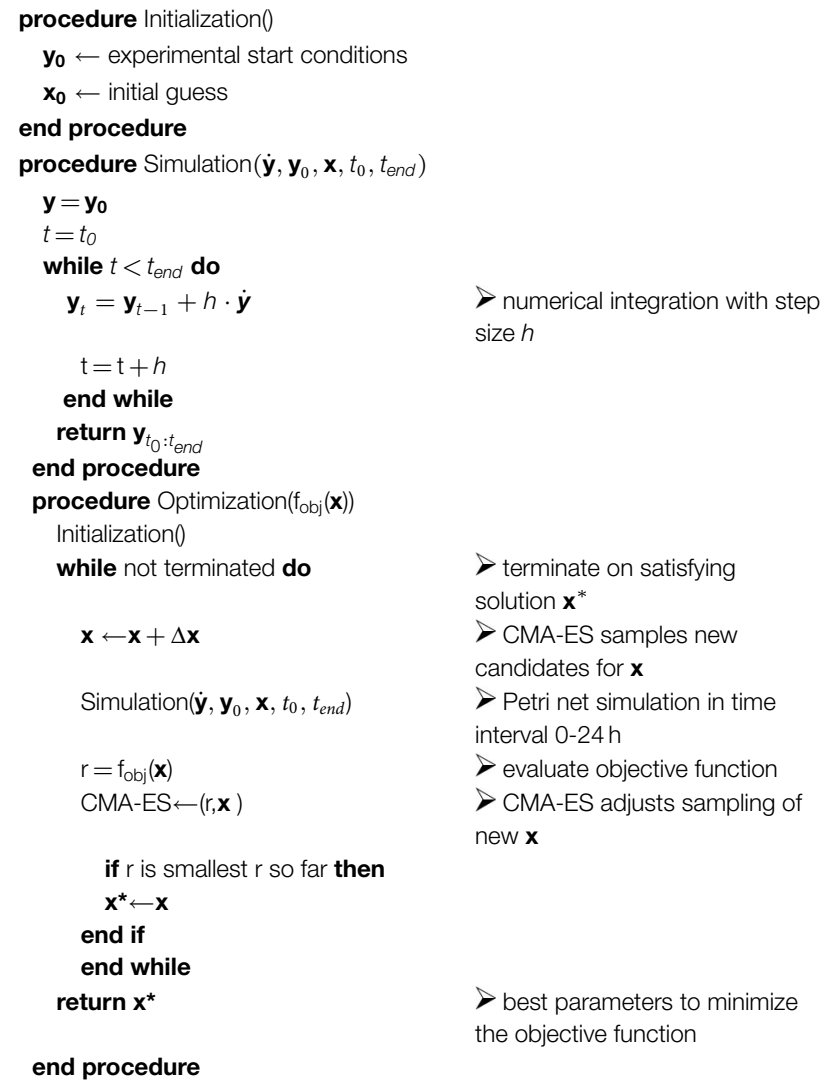

$>$ numerical integration with step size $h$

$>$ terminate on satisfying solution $\mathbf{x}^{*}$

$>$ CMA-ES samples new candidates for $\mathbf{x}$

$>$ Petri net simulation in time interval 0-24 $\mathrm{h}$

$>$ evaluate objective function $>$ CMA-ES adjusts sampling of new $\mathbf{x}$

$>$ best parameters to minimize the objective function

inflammatory response and mitochondrial dysfunction $(11,12)$. The role of HS in cardiac apoptosis and necroptosis, however, is unknown.

The use of in vitro experiments to investigate pathophysiological processes is complex, time-consuming, and expensive. A new and very promising solution to overcome these shortcomings of "classical" experimental techniques aimed at understanding biology is the modeling of system biological processes using

computer-based methods (16-18). The access to data, however, is very limited in in vitro studies, facing a small data problem. Therefore, we established a methodology that involves expert knowledge in the modeling processes combined with the optimization of the unknown parameters using evolutionary algorithms (19-21). This family of algorithms is inspired from biological evolution and is adequate for optimization problems that lack full mathematical formalization between the tunable parameters and optimization objectives (22). Introduced by Carl Adam Petri in 1962, a Petri net is a simple graph that is built from places and transitions, which are interconnected by weighted arcs. In systems biology, places correspond to the measured amount of a substrate, transitions model changes in substrate [by ordinary differential equations (ODEs)], while weighted arcs model the influence of the specific transition on a place or vice versa. Petri nets are intuitive and offer both visualization and a mathematical formalism. Nevertheless, they are a very powerful tool for modeling complex (i.e., biological) processes and are, hence, emerging as promising and powerful tools in systems biology (23-26). As Petri nets have different abstraction levels, it is possible to model different biological processes (27). In this study, models were adapted and verified with available in vitro data sets. The structure of Petri nets can be extended to a system of ODE modeling the kinetic information. This allows us to extract a system of ODEs by defining transition functions between the substrates that are modeled by commonly used mass action kinetics $(24,28)$. Their application to biological processes, such as signal pathways, may have the potential to facilitate the generation of valuable data, which otherwise (with classical techniques) would be expensive and time-consuming to generate.

Thus, the aim of the present study was (i) to investigate if HS induces apoptosis or necroptosis in cardiomyocytes and (ii) to evaluate the signaling pathways involved. Additionally, we aimed to develop a proof-of-concept study for using Petri nets to simulate the missing data (different concentrations and time-points) by using evolutionary optimization to optimize the kinetic parameters and involving expert knowledge to model the structure of the network. Using this novel Medical-In silico approach, our 
$\mathrm{A}$ totERK $1 / 2 \rightleftharpoons \overline{=}={ }_{42 \mathrm{kDa}}^{44 \mathrm{ka}}$
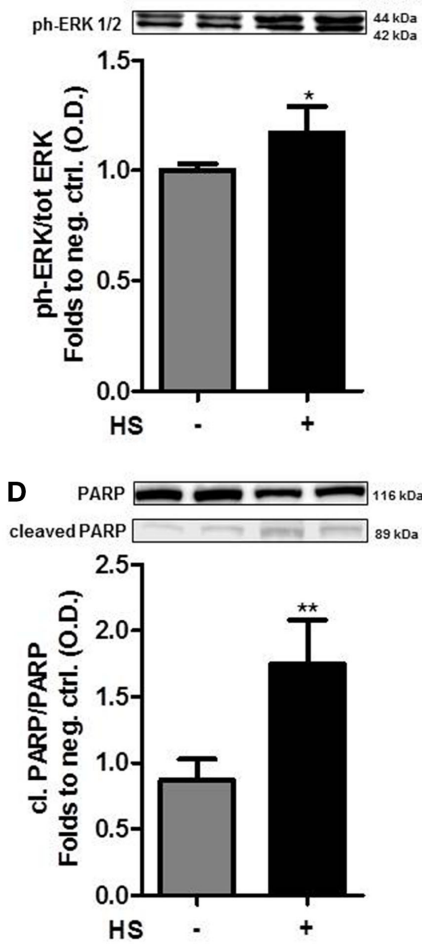
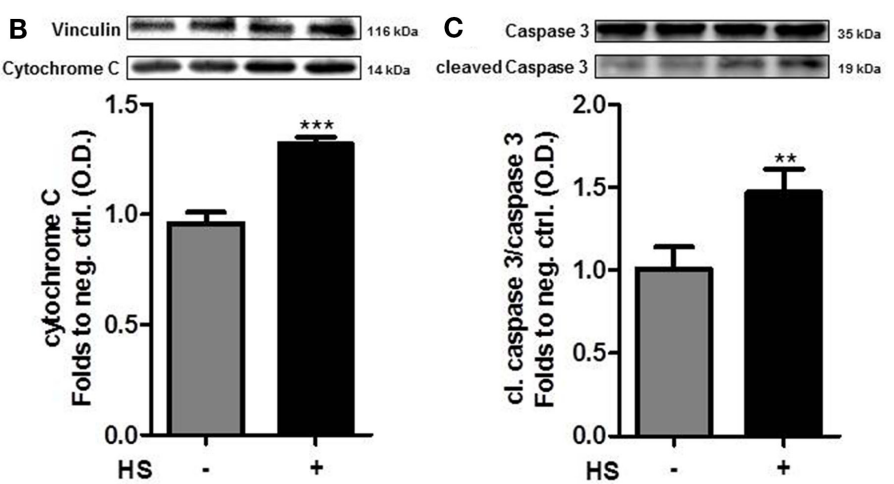

E

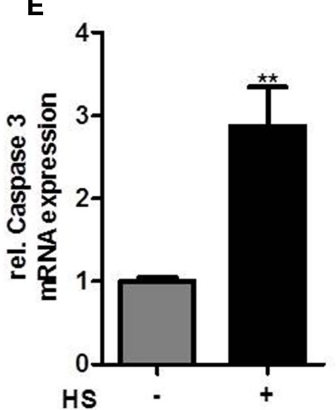

$\mathbf{F}$

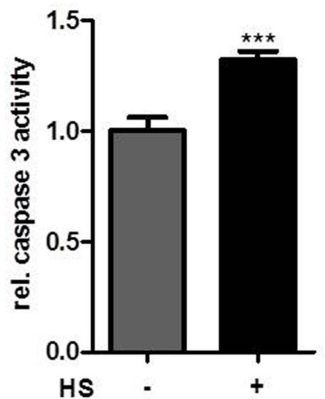

FIGURE 2 | Heparan sulfate induces a pro-apoptotic pathway. $\mathrm{HL}-1$ cells exposed to $10 \mu \mathrm{g} / \mathrm{ml} \mathrm{HS}$ for $16 \mathrm{~h}$ showed a significant increase in protein expression of (A) phospho-ERK 1/2, (B) cytochrome C, (C) cleaved PARP, and (D) cleaved caspase 3, compared to unstimulated cells. Protein expression was normalized to unstimulated cells. (E) Relative mRNA expressions of HL-1 cells exposed to HS were analyzed by quantitative real-time PCR, compared to unstimulated cells. Caspase 3 mRNA expression was normalized to reference gene S7 and unstimulated cells. (F) Relative caspase 3 activity of cardiomyocytes exposed to HS, compared to unstimulated cells. The data represent the mean \pm SD of triplicate samples for three independent experiments. HS, heparan sulfate; PARP, poly-(ADP-ribose) polymerase; $p$-ERK, phospho-extracellular signal-regulated kinase; statistical significance was performed by using unpaired $t$-test. ${ }^{*} p<0.05$; ${ }^{* \star} p<0.01$, and ${ }^{\star * \star} p<0.001$ vs. unstimulated cells.

data show (i) that HS induces necroptosis in cardiomyocytes by phosphorylation (activation) of RIP3 and (ii) indicate that this proof-of-concept is a first step toward simulating the extent of activated components in the pro-apoptotic pathway induced by HS with only a small data set gained from the in vitro experiments by using machine learning algorithms.

\section{MATERIALS AND METHODS}

\section{Cell Culture}

As described previously (12, 29-31), HL-1 cells (murine cardiomyocytes) were cultured in $10 \mathrm{~cm}$ plates coated with a gelatin/fibronectin solution [ $5 \mathrm{mg} / \mathrm{L}$ fibronectin (Sigma, Munich, Germany), $0.02 \%(w / v)$ gelatin (Sigma)]. Cells were cultivated in Claycomb medium (Sigma) and incubated under an atmosphere of $5 \% \mathrm{CO}_{2}$ and $95 \%$ air at $37^{\circ} \mathrm{C}$. The medium was supplemented with $50 \mathrm{ml}$ fetal calf serum (10\%, Biochrom, Berlin, Germany), $5 \mathrm{ml}$ norepinephrine ( $0.1 \mathrm{mM}$, Sigma), $5 \mathrm{ml}$ L-glutamine (2 mM, Sigma-Aldrich, Munich, Germany) and $5 \mathrm{ml}$ penicillin/streptomycin (Invitrogen, Carlsbad, CA, USA).

\section{Cell Stimulation}

Cardiomyocytes were exposed to $10 \mu \mathrm{g} / \mathrm{ml} \mathrm{HS}$ (Amsbio, Abingdon, UK) for $16 \mathrm{~h}$. We used unstimulated cells as a negative control, and the cells exposed to $2.5 \mu \mathrm{M}$ staurosporine as a positive control (AppliChem, Darmstadt, Germany).

\section{RNA Extraction and qPCR}

RNA was isolated using the Trizol reagent, as described earlier (12, $30,31)$. The following primers were used to analyze the relative mRNA expression of caspase 3 and TNF- $\alpha$ in the quantitative real-time PCR (StepOnePlus Real-Time PCR System; Thermo Fisher Scientific, MA, USA): caspase 3, $5^{\prime}$ CCAACCTCAGAGAGACATTC $3^{\prime}$ (for) and 5' TTTCGGCTTTCCAGTCAGAC $3^{\prime}$ (rev) and TNF- $\alpha, 5^{\prime}$ TCCCCAAAGGGATGAGAAG $3^{\prime}$ (for) and $5^{\prime}$ GCACCACTAGTTGGTTGTC $3^{\prime}$ (rev). S7 was used as the reference gene: $5^{\prime}$ GGTGGTCGGAAAGCTATCA 3' (for) and 5' AAGTCCTCAAGGATGGCGT 3' (rev). Relative quantification was performed by using Microsoft Excel (Microsoft, Washington, DC, USA).

\section{Western Blot Analysis}

Cardiomyocytes were washed with PBS and lysed using Triton lysis buffer [300 mM NaCl (Roth, Karlsruhe, Germany), $20 \mathrm{mM}$ TRIS (pH 7.4) (Merck, MA, USA), 1\% Triton-X100 (SigmaAldrich), 200 mM PMSF (Roth), 1 mM DTT (Gerbu, Heidelberg, Germany), $2 \mathrm{mg} / \mathrm{ml}$ leupeptin (AppliChem), and $1 \mathrm{mg} / \mathrm{ml}$ pepstatin (AppliChem)] (12, 30). After $30 \mathrm{~min}$ on ice, the cells were 

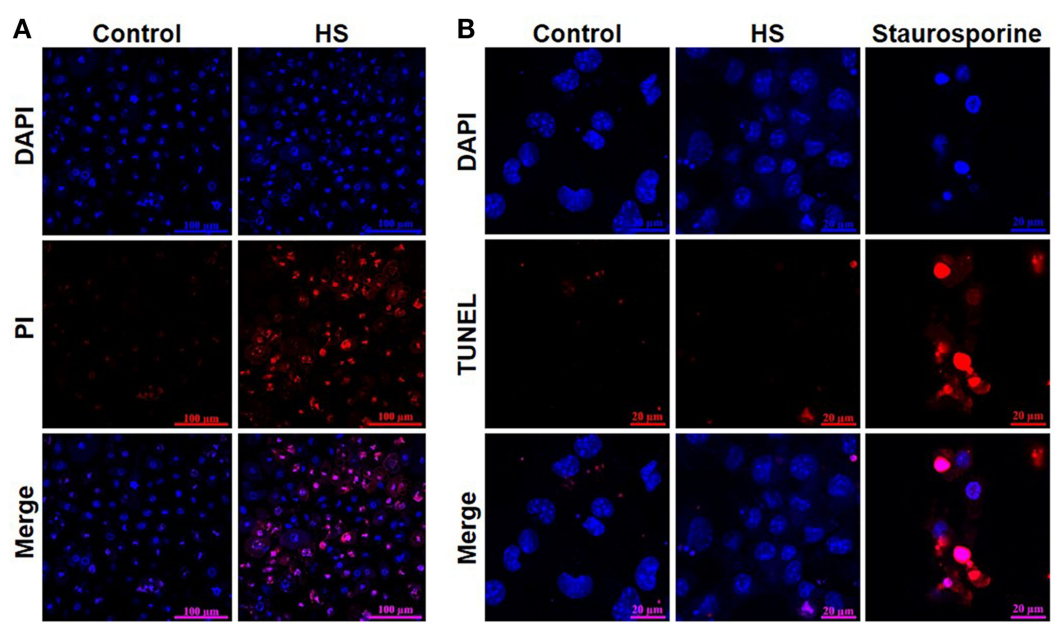

FIGURE 3 | Heparan sulfate induces cell death but no apoptosis in cardiomyocytes. (A) HL-1 cardiomyocytes exposed to $10 \mu \mathrm{g} / \mathrm{ml}$ of HS for $16 \mathrm{~h}$ were stained with Hoechst and $\mathrm{PI}$ and compared to unstimulated cells (control) and analyzed with confocal live-cell imaging. Hoechst represent the cell nuclei (blue) and PI the death cells (red). (B) HL-1 cardiomyocytes exposed to HS for $16 \mathrm{~h}$ or $2.5 \mu \mathrm{M}$ staurosporine were stained with DAPI and TUNEL and compared to unstimulated cells. DAPI represent the cell nuclei (blue) and TUNEL the apoptotic cells (red). HS, heparan sulfate; DAPI, 4' ,6-diamidino-2-phenylindole; PI, propidium iodide; TUNEL,

TdT-mediated dUTP-biotin nick end labeling.

centrifuged for $10 \mathrm{~min}$ at $4^{\circ} \mathrm{C}$ and $20,800 \times g$. The supernatant was transferred to a new tube, and the protein concentrations were determined by the Bradford method (Roti-Quant, Roth). Proteins were separated by $12 \%$ sodium dodecyl sulfate polyacrylamide gel electrophoresis. Gel electrophoresis was carried at $120 \mathrm{~V}$. Separated proteins were transferred onto a polyvinylidene diflouride membrane. After blocking, membranes were incubated with specific primary antibodies against caspase 3 (Cell Signaling, Danvers, MA, USA), poly-(ADP-ribose) polymerase (PARP) (Cell Signaling), extracellular signal-regulated kinase (ERK) $1 / 2$ (Cell Signaling), phospho-ERK 1/2 (Cell Signaling), cytochrome C (Cell Signaling), RIP3 (Bio-Rad), phospho-RIP3 (Ser232) (Abcam), MLKL (Cell Signaling), phospho-MLKL (Ser345) (Cell Signaling), and vinculin (Sigma). After incubation with a second antibody for $1 \mathrm{~h}$ at room temperature, proteins were detected with the ECL Prime Western Blotting Detection Reagent (GE Healthcare, Uppsala, Sweden) and the LAS-4000-System (Fujifilm, Tokyo, Japan).

\section{TdT-Mediated dUTP-Biotin Nick End Labeling (TUNEL)}

This method was first described in 1992 by Gavrieli et al. to detect apoptotic cells (32). HL-1 cells were grown on glass coverslips coated with gelatin/fibronectin and exposed to HS or staurosporine for $16 \mathrm{~h}$, respectively. After $16 \mathrm{~h}$, the cells were washed three times with PBS and fixed with $500 \mu \mathrm{l}$ of $4 \%$ PFA (Sigma-Aldrich) for $1 \mathrm{~h}$ at room temperature. Next, the cells were permeabilized $(0.1 \%$ Triton X-100, $0.1 \%$ sodium citrate) for $2 \mathrm{~min}$ on ice. Cells were labeled with $5 \mu \mathrm{l}$ of TUNEL-Enzyme and $45 \mu \mathrm{l}$ of TUNEL-Label Solution (In situ Cell Detection Kit TMR red, Roche, Mannheim, Germany) for $1 \mathrm{~h}$ at $37^{\circ} \mathrm{C}$ in the dark. Cells only labeled with $45 \mu \mathrm{l}$ of TUNEL-Label Solution were used as a negative control. After washing, the nuclei were stained with $15 \mu \mathrm{l}$ of $4^{\prime}$,6-diamidino-2-phenylindole. LSM 710 confocal microscope
(Zeiss, Oberkochen, Germany) was used for detection and further analysis.

\section{Fluorescence Flow Cytometry}

Stimulated cardiomyocytes were washed three times with PBS on ice and harvested with a scraper. After centrifugation at $4^{\circ} \mathrm{C}$ and $500 \times g$ for $5 \mathrm{~min}$, the supernatant was discarded, and the cells were resuspended in binding buffer, stained with Annexin V (\#550474) and 7-AAD (\#559925), and then analyzed by Fortessa LSR (all BD Biosciences, NJ, USA). Unstained cells were used for gating the cells (upper left panel, Figure 4). The Annexin V and 7 -AAD plots from the gated cells show the populations corresponding to viable cells (both Annexin $\mathrm{V}$ and 7-AAD negative, Gate I), early apoptotic cells (Annexin V positive and 7-AAD negative, Gate II), late apoptotic cells (both Annexin V and 7AAD positive, Gate III), and necrotic cells (Annexin V negative and 7-AAD positive cells, Gate IV).

\section{Cell Vitality}

Cells were grown on $\mu$-slide 8-wells (IBIDI, Martinsried, Germany) to analyze cell viability. Cells stimulated for $16 \mathrm{~h}$ were washed with PBS and stained with propidium iodide (PI, BD Biosciences) and $5 \mu \mathrm{g} / \mathrm{ml}$ Hoechst (H3570, Invitrogen). Cell viability of cardiomyocytes was detected with live-cell imaging at $37^{\circ} \mathrm{C}$ and $5 \% \mathrm{CO}_{2}$ in an incubator at an LSM 710 confocal microscope. ImageJ software was used to count the amount of dead cells.

\section{Statistical Analysis of the In vitro Measurements}

The statistical analysis and the graphs of the relative protein expression were performed with GraphPad Prism 5 (GraphPad Inc., San Diego, CA, USA). An unpaired $t$-test or one-way ANOVA followed by the Bonferroni test was used for multiple 
comparisons with a significance level of $p<0.05$. The data represent the mean $\pm S D$ for three independent experiments performed in triplicates.

\section{Medical-In silico Model}

The measured in vitro data formed the basis for utilizing specific machine learning methods. The model was designed as a continuous Petri net (33). This allowed us to use the model for a computational prediction of involved signaling pathways describing the induced necroptosis in cardiomyocytes exposed to a given amount and time of HS. The structure of the Petri net was constructed analogous to the current understanding of the pathophysiological process of necroptosis. The mass action kinetics was implemented to model the pathophysiological process. This kinetics was represented by ODEs in the transitions of the Petri net (24). These ODEs were parameterized, where the parameters are adapted based on evolutionary optimization. As typical for results gained from in vitro measurements, our approach targeted the small data challenge. Thus, we optimized the parameters of the ODEs in order to minimize the mean square error of the given data samples to the simulated data.

The designed model is rather abstract and represents only a part of entire and complex pathophysiological process of apoptosis or necrosis. The model was constructed and optimized as follows (Figure 7):

I. The pathophysiological process was comprehensively defined (Figure 7).

II. Analog to the preceding description, the Petri net structure was derived.

III. ODEs were extracted from the structure of the Petri net. The transition functions between the substrates were based on the mass action kinetics (Table 1).

- The parameters of the kinetics (Figure 8) were optimized in a way that the simulated data fitted the measured data according to a minimum mean square error approach.

- The output of the model was simulated based on the Matlab ODE solver (34) (Algorithm 1).

IV. Based on the system of ODEs, an optimization problem was formulated that incorporates the in vitro measurements.

V. The ODEs were parameterized by Covariance Matrix Adaption-Evolutionary Strategy, an algorithm that minimizes the objective function $(35,36)$.

VI. A visualization of the time course was simulated by numerical integration from the parameterized ODEs.

VII. Finally, the model was analyzed and either accepted or previous design steps were refined.

\section{Optimization Process}

The optimization is mainly based on data acquired from in vitro experiments (39). Each protein and mRNA expression of the pathway is measured for a specific initial amount of induced HS after a specific time. For a mathematical formulation of the conducted optimization problem, i.e., fitting the model parameters to the experimental data, we defined a real valued observation function $g(\theta)$ that provides the experimental data to the algorithm. Since each place in the Petri net corresponds to a component of the signaling pathway, $g(\theta)$ is equivalent to a noisy measurement of place $p$ within the model at time $t$ and initial state $s(40,41)$. Each in vitro measurement is defined by the triple $\theta=(p, t, s)$, so that $\Theta$ is the set of all in vitro measurements. For instance, cleaved PARP is measured after $16 \mathrm{~h}$ within an in vitro experiment that was carried out for $10 \mu \mathrm{g} / \mathrm{ml}$ induced HS. Since cleaved PARP corresponds to place $p_{4}$ in our model, the exemplary measurement is defined by $\theta=\left(p=p_{4}, t=16 h, s=10 \mu \mathrm{g} / \mathrm{ml} \mathrm{HS}\right)$. To fit the model's parameters, only a subset of all measurements $\Theta$ was used. This training set is denoted by $\Theta_{T}$. The remaining measurements are used for later validation of the acquired model. Following these definitions, the square errors between in vitro measurements and model simulation $y(\theta ; x)$ (where $x$ denote the parameter vector of the petri net model) are formulated by

$$
f_{0}(x)=\sum_{\theta \in \Theta_{T}}|g(\theta)-y(\theta ; \boldsymbol{x})|^{2} .
$$

In order to satisfy the biological constraints, i.e., magnitudes of relative protein expressions, by the model, we added penalties to the objective function. Thereby, the model's protein expressions are bound into reasonable magnitudes. To achieve this, the model should fulfill the upper bounds $u_{p}$ and lower bounds $l_{p}$ for simulated values of places $p$. The penalties are weighted by nonnegative $\lambda_{l}, \lambda_{u}$ and evaluated for all simulated times, places, and initial HS amounts, called $\Theta_{S}$, by

$$
\begin{aligned}
f_{u}(\boldsymbol{x}) & =\lambda_{u} \sum_{\theta \in \Theta_{S}}\left|\left(y(\theta ; \boldsymbol{x})-u_{p}\right)^{+}\right|^{2} \\
f_{l}(\boldsymbol{x}) & =\lambda_{l} \sum_{\theta \in \Theta_{S}}\left|\left(l_{p}-y(\theta ; \boldsymbol{x})\right)^{+}\right|^{2} .
\end{aligned}
$$

The penalties, which are added to the objective function, allow us to enforce the realistic modeling of the biological behavior. This leads to more realistic parameters. Furthermore, all parameters are restricted to positive real values $\left(x_{l b}>0\right)$ with a limited magnitude $\left(x_{\mathrm{ub}}<\infty\right)$. All these assumptions result in the following optimization problem:

$$
\begin{aligned}
& \mathbf{x}^{\star}=\underset{\mathbf{x}}{\arg \min } f_{\mathrm{o}}(\mathbf{x})+f_{u}(\mathbf{x})+f_{1}(\mathbf{x}) \\
& \text { subject to } \mathbf{x}_{l b}<\mathbf{x}<\mathbf{x}_{u b} .
\end{aligned}
$$

The above mentioned problem is solved with the CMAES optimizer $(22,35,36)$. By this, the optimal set of parameters, $\mathrm{x}^{*}$ that minimizes the objective function is utilized for numerical simulation of the model and time course prediction. The optimization routine is given in the listing of Algorithm 1. The source code for the presented procedure is made available on GitHub (https://github.com/Medical-In-silico/In-silico-Heparan-sulfateinduced-necroptosis-in-murine-cardiomyocytes, accessed February 4,2018 ).

\section{RESULTS}

\section{HS Activates a Pro-Apoptotic Signal Cascade in Cardiomyocytes}

The exposure of cardiomyocytes to HS for $16 \mathrm{~h}$ resulted in a significant increase in the phosphorylation of ERK $1 / 2$ on 
unstained

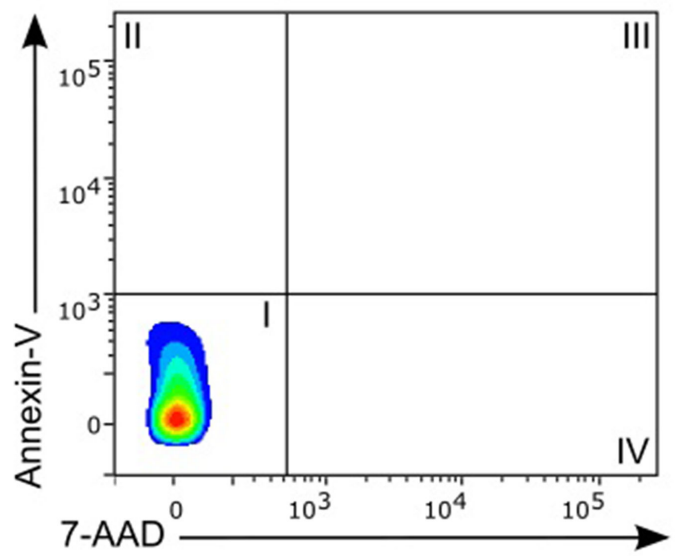

HS

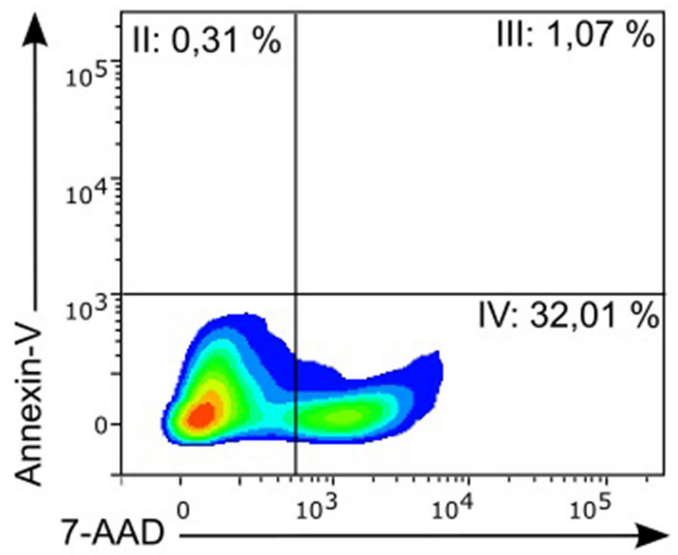

control
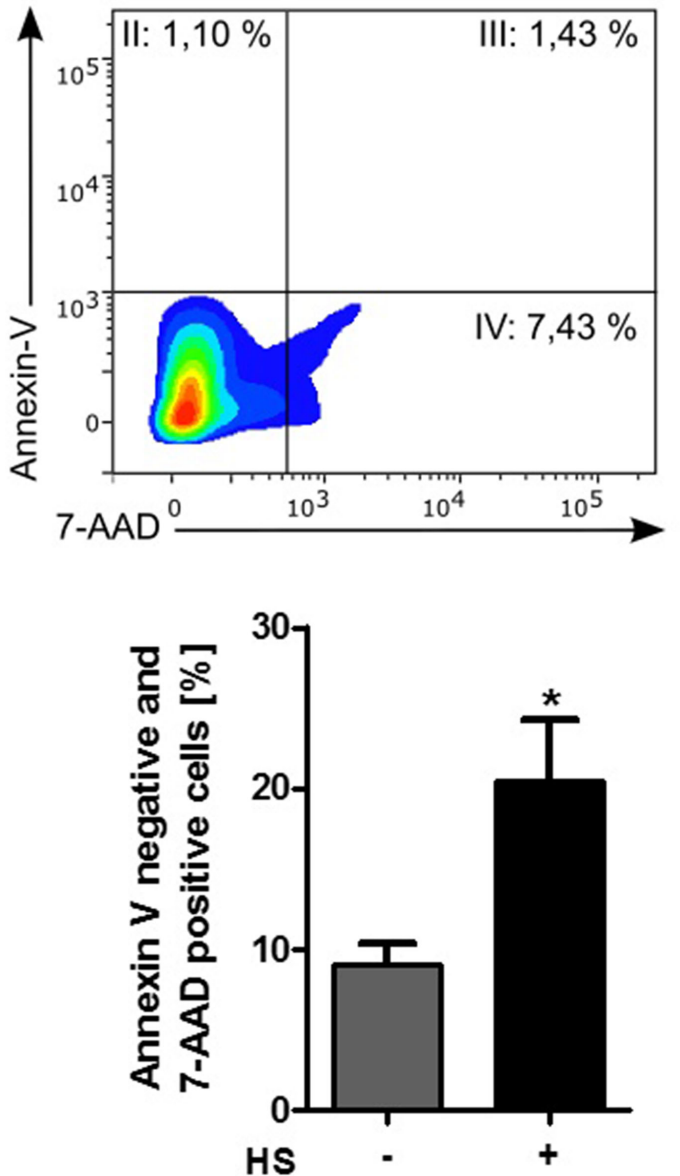

FIGURE 4 | Cell death analysis by using FACS. HL-1 cells exposed to $10 \mu \mathrm{g} / \mathrm{ml}$ of HS and unstimulated cells (control) were stained with Annexin V and 7-AAD and then analyzed by fluorescence-based flow cytometry. Unstained cells were used for gating the cells. The Annexin $V$ and 7 -AAD plots from the gated cells show the populations corresponding to viable cells (both Annexin $V$ and 7-AAD negative, Gate I), early apoptotic cells (Annexin V positive and 7-AAD negative, Gate II), late apoptotic cells (both Annexin V and 7-AAD positive, Gate III), and necrotic cells (Annexin V negative and 7-AAD positive cells, Gate IV). The bar chart represents the percentage (mean \pm SD) of necrotic cells (Annexin V-negative and 7-AAD-positive cells) in unstimulated (control) and HS-treated HL-1 cells, respectively. Triplicate samples for three independent experiments are shown. HS, heparan sulfate; ${ }^{*} p<0.05$ control vs. HS-treated HL-1 cells.

Thr202/Tyr204 ( $p<0.05$; Figure 2A) and cytochrome C levels $(p<0.001$; Figure 2B). HS stimulation also resulted in a significant activation (cleavage) of caspase 3 and inactivation of PARP compared to unstimulated cells $(p<0.01 ; p<0.05$; Figures 2C,D). Furthermore, expression of caspase 3 mRNA $(p<0.001$; Figure 2E) and relative caspase 3 activity were significantly increased ( $p<0.001$; Figure 2F).

\section{HS Induces Necrosis, but Not Apoptosis in Cardiomyocytes}

A total of 4,262 unstimulated cells and 4,243 HS-stimulated cardiomyocytes were examined with confocal live-cell imaging. The amount of dead cells was quantified by PI staining. Unstimulated cells showed a cell viability of $96.15 \%$; however, cardiomyocytes exposed to HS showed a cell viability of $69.86 \%$ (Figure 3A). We next investigated the number of apoptotic cells. Notably, no apoptotic cells could be detected after exposure to HS, indicated by the lack of TUNEL-positive cells. In contrast, the stimulation with staurosporine for $16 \mathrm{~h}$ (as positive control) resulted in a significant amount of TUNEL-positive (apoptotic) cells (Figure 3B). Having shown that the exposure of HS resulted in a significant amount of cell death but no induction of apoptosis, we next investigated the amount of necrotic as well as apoptotic cells by using FACS. Unstained cells were used for gating the cells (upper left panel, Figure 4). The Annexin V and 7-AAD plots from the gated cells show the populations corresponding to viable cells (both Annexin V and 7-AAD negative, Gate I), early apoptotic cells (Annexin $\mathrm{V}$ positive and 7-AAD negative, Gate II), late apoptotic cells (both Annexin V and 7-AAD positive, Gate III), and necrotic cells (Annexin V negative and 7-AAD positive cells, Gate IV). Untreated cells (control) showed that the majority of cells were viable and non-apoptotic/necrotic (Gate I, upper right panel). In contrast to untreated cells $(9.02 \pm 1.34 \%$ Annexin $\mathrm{V}$-negative and 7-AAD positive cells), the exposure of HL-1 cells to HS resulted in $20.40 \pm 3.91 \%$ Annexin V-negative and 7-AAD-positive cells (Gate IV, upper right and lower left panel, $p<0.05)$. 

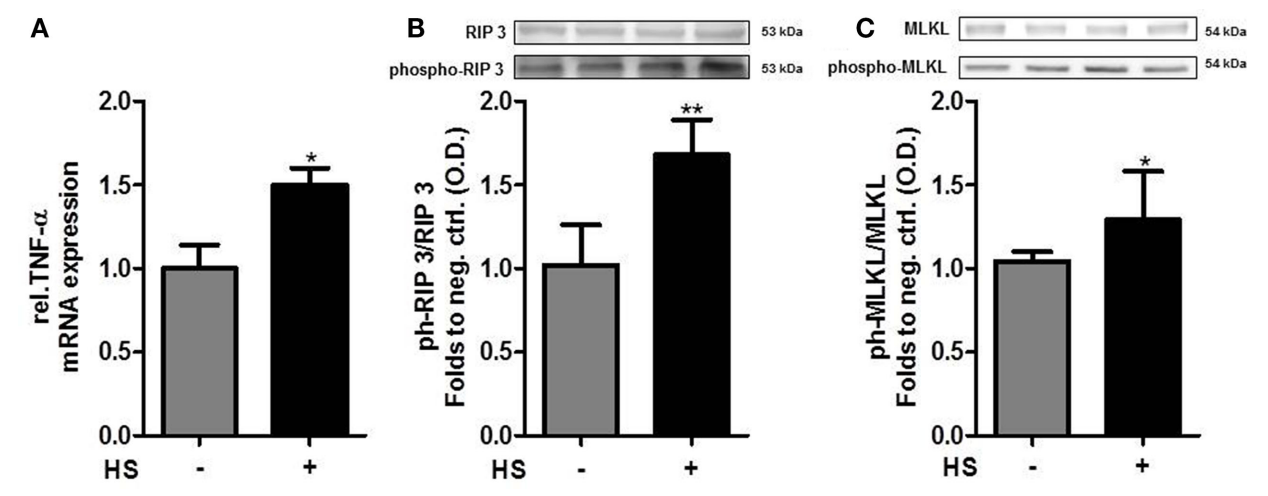

FIGURE 5 | Heparan sulfate induces necroptosis in cardiomyocytes. (A) Relative caspase 3 mRNA expressions of HL-1 cells exposed to HS for 16 h were analyzed by quantitative real-time PCR, compared to unstimulated cells. Expression was normalized to reference gene S7 and normalized to unstimulated cells. (B) HL-1 cells exposed to $10 \mu \mathrm{g} / \mathrm{ml} \mathrm{HSs}$ for $16 \mathrm{~h}$ showed a significant increase in phosphorylation of RIP3 and (C) MLKL compared to unstimulated cells. Protein expression was normalized to unstimulated cells. The data represent the mean \pm SD of triplicate samples for three independent experiments. HS, heparan sulfate; RIP, receptor-interacting protein; MLKL, mixed lineage kinase domain-like; TNF- $\alpha$, tumor necrosis factor alpha; ${ }^{\star} p<0.05$, ${ }^{\star \star} p<0.01$ vs. unstimulated cells.

\section{HS Induces Necroptosis, a Programmed form of Necrosis, in Cardiomyocytes}

Cardiomyocytes exposed to HS for $16 \mathrm{~h}$ showed a significant increase in the relative expression of TNF- $\alpha$ mRNA, compared to unstimulated cells $(p<0.05$; Figure 5A). The exposure of cardiomyocytes to HS also resulted in a significant increase in the phosphorylation of RIP3 on Ser232 ( $p<0.01$; Figure 5B). Furthermore, cardiomyocytes exposed to HS showed a significant increase in the phosphorylation of MLKL on Ser345 $(p<0.05$; Figure 5C) compared to unstimulated cells, indicating that activated MLKL and RIP3 form a necrosome.

\section{Time Course of PAPR Inactivation}

In order to use the new Medical-In silico approach, data from in vitro experiments of one component of the signal pathway induced by HS at different time points and concentrations were necessary. Thus, we generated in vitro a time course of the relative protein expression of cleaved PARP in cardiomyocytes exposed to 5,10 , and $20 \mu \mathrm{g} / \mathrm{ml} \mathrm{HS}$ for $4,8,16$, and $24 \mathrm{~h}$, respectively, using Western blot analysis. The exposure of cardiomyocytes to either 5 , 10 , or $20 \mu \mathrm{g} / \mathrm{ml} \mathrm{HS}$ resulted in an increase in PARP inactivation, with a peak of relative PARP inactivation after the exposure of cardiomyocytes to $20 \mu \mathrm{g} / \mathrm{ml} \mathrm{HS}$ for $16 \mathrm{~h}$ (Figure 6).

\section{Simulated Time Course of Involved Components in Apoptosis and Necroptosis Pathway}

Next, we developed a proof-of-concept study for using Petri nets to simulate the missing data (different concentrations and timepoints) by using evolutionary optimization to optimize the kinetic parameters and involving expert knowledge to model the structure of the network. Using the measured time course of cleaved PARP together with the results of the other measured components in cardiomyocytes exposed to $10 \mu \mathrm{g} / \mathrm{ml} \mathrm{HS}$ for $16 \mathrm{~h}$, we simulated the results for the missing time points and concentrations (Figure 9). Three dose-response curves over time were simulated [5 (green), 10 (blue), and 20 (purple) $\mu \mathrm{g} / \mathrm{ml}$ of HS].

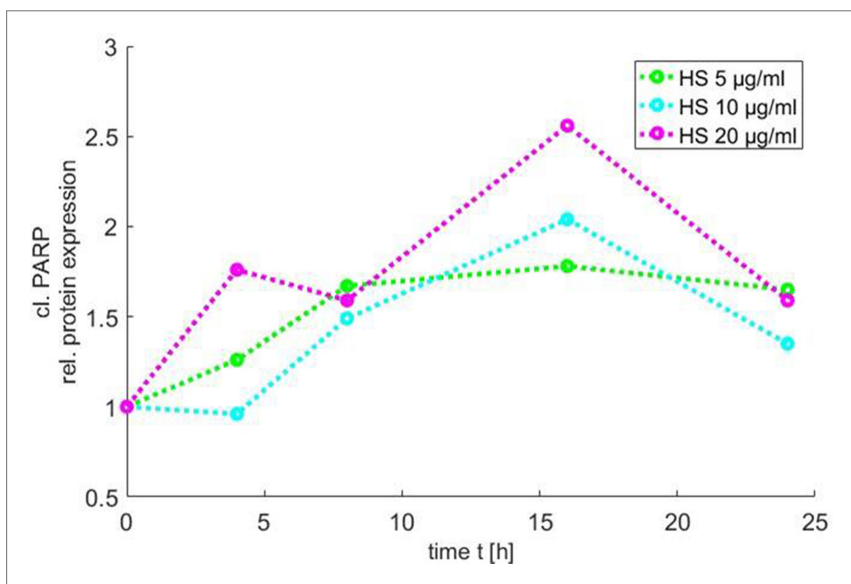

FIGURE 6 | Time course of PARP inactivation. The relative PARP inactivation was analyzed by using Western blot and detected after $4,8,16$, and $24 \mathrm{~h}$ after treatment of cardiomyocytes with 5,10 , or $20 \mu \mathrm{g} / \mathrm{ml} \mathrm{HS}$, respectively. HS, heparan sulfate; PARP, poly-(ADP-ribose) polymerase.

The simulation showed an increase in phosphorylation of ERK $1 / 2$ in the first $2 \mathrm{~h}$ for all three HS concentrations. After $3 \mathrm{~h}$, the phosphorylation of pERK $1 / 2$ reached its maximum. The highest phosphorylation of pERK $1 / 2$ could be detected after the exposure of $20 \mu \mathrm{g} / \mathrm{ml}$ of HS. After $3 \mathrm{~h}$, a decrease in phosphorylation of pERK $1 / 2$ was observed that reached baseline after $24 \mathrm{~h}$ (Figure 9A). The simulation also showed an increase in relative protein expression for cytochrome $\mathrm{C}$ with peak values being observed after $4 \mathrm{~h}$ (Figure 9B). Figure 9C shows the simulation of the activation of caspase 3 over time. The protein expression of the cleaved (activated) caspase 3 continuously increased until its peak at $8 \mathrm{~h}$ (independent of the investigated concentration) (Figure 9C). The simulated time course for cleaved PARP was (as expected) very similar to the measured cleaved PARP values (Figure 6), as more measured data were used for this parameter to train and validate the model. The simulated relative protein expression of cleaved PARP increased and peaked after $12 \mathrm{~h}$. The simulation showed a slow decrease in the relative cleaved PARP 


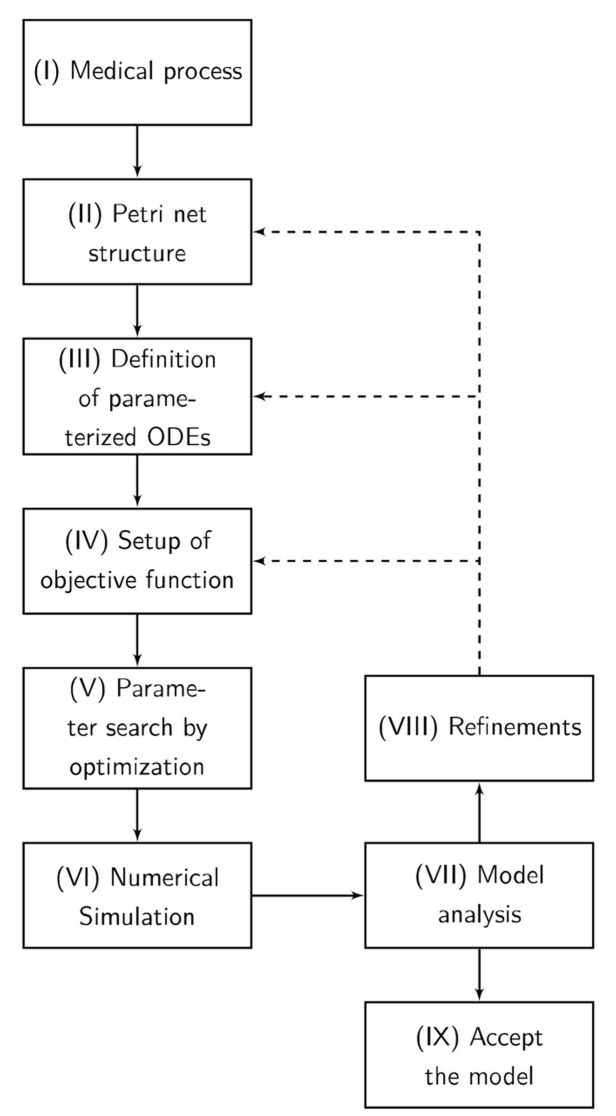

FIGURE 7 | The flowchart presents our Medical-In silico approach. It shows all the undertaken processes from the pathophysiological process to the final model. (I) The pathophysiological process is comprehensively defined. (II) Analog to the preceding description, the Petri net structure is derived. (III) Ordinary differential equations (ODEs) are extracted from the structure of the Petri net. (IV) Based on the system of ODEs, an optimization problem is formulated incorporating in vitro measurements. We might enhance the objective function with some penalty functions that drive the model into biological boundaries. ( $\mathrm{N}$ ) The ODEs are parameterized by an evolutionary strategy algorithm that minimizes the objective function. (VI) A visualization of the time course is simulated by numerical integration from the parameterized ODEs. (VII) Finally, the model is analyzed and either accepted or previous design steps are refined.

protein expression after the peak values and did not reach the baseline within the simulated time (Figure 9D). The phosphorylation of RIP3 showed its maximum after $14 \mathrm{~h}$ with only a slight decrease for all three concentrations after reaching the peak values (Figure 9E). The simulation indicated an increase in the relative TNF- $\alpha$ mRNA expression during the first $8 \mathrm{~h}$, which did not reach baseline values within the simulated time (Figure 9F).

\section{DISCUSSION}

Trauma and sepsis cause tissue injury, resulting in the release of DAMPs that are able to induce a pro-inflammatory signal cascade and the release of pro-inflammatory cytokines such as TNF- $\alpha$ (42). Several signaling pathways involved in apoptosis and necroptosis are linked to trauma- or sepsis-associated cardiomyopathy. However, the underling causative factors are still

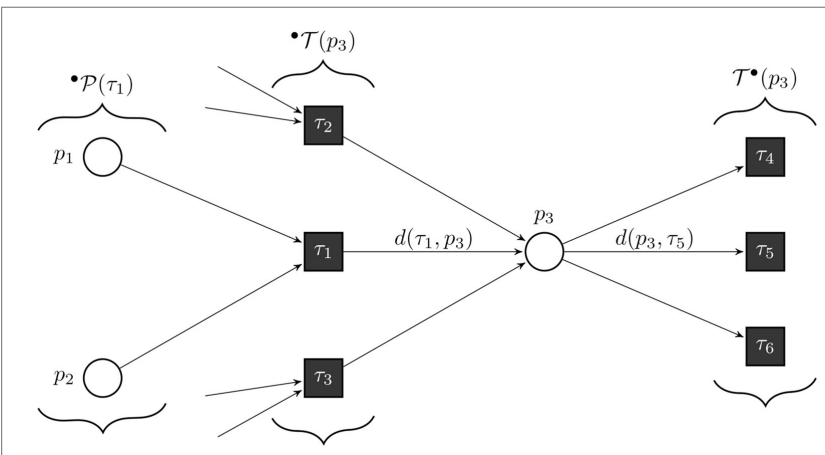

FIGURE 8 | Formal definition of a continuous Petri net (CPN). Our model is a CPN. CPNs can be represented equivalently by ordinary differential equations and, thus, inherit dynamical behavior. The exemplary CPN shows our notation: ${ }^{\circ} T(p)$ is the set of all incoming transitions of place $p . T^{\circ}(p)$ is the set of all outgoing transitions of place $p .{ }^{\circ} P(\tau)$ is the set of all places with an edge directed to transition $\tau$. With this notation, we define the transition functions by $\frac{\partial y(p)}{\partial t}=\sum_{\tau \in \mathcal{T}(p)} d(\tau, p) v(\mathcal{T})-\sum_{\tau \in \mathcal{T} \bullet(p)} d(p, \mathcal{T}) v(\mathcal{T})$. In the case of general mass action kinetics (38), the transitions are given by $v(\mathcal{T})=x(\mathcal{T}) \cdot \prod_{p \in \bullet \mathcal{P}(\mathcal{T})} y(p)^{d(\tau, p)}$, where $x(\tau)$ are the kinetic rates.

unknown. Using a novel Medical-In silico approach, this study shows (i) that HS induces necroptosis in cardiomyocytes by phosphorylation (activation) of RIP3, (ii) suggest that HS may have the potential as a therapeutic target in trauma- or sepsis-associated cardiomyopathy, and (iii) indicate that this proof-of-concept is a first step toward simulating the extent of activated components in the pro-apoptotic pathway induced by HS with only a small data set gained from the in vitro experiments by using machine learning algorithms.

\section{Activation of the Pro-Apoptotic Signaling Cascade by HS}

Cell death caused by inflammation can be mediated by two processes: necrosis and apoptosis. It is well known that both, necrosis and apoptosis, lead to a loss of intact cardiomyocytes in cardiovascular diseases (10). Apoptosis represents a type of programmed cell death that is regulated by caspases cascade (8) and activated by two different pathways: the extrinsic and the intrinsic pathway (43). In the intrinsic pathway, the pro-apoptotic signaling cascade is proceeded by the release of mitochondrial cytochrome C. ERK $1 / 2$ belong to the family of mitogen-activated protein kinases (MAPKs) which are involved in different cell processes and cell death (44). Phosphorylation and, thus, activation of ERK $1 / 2$ by extracellular stimuli (such as DAMPs) results in the release of cytochrome C (45). Indeed, our data indicate that the exposure of HS to cardiomyocytes results in an increased expression of phosphorylation of ERK 1/2 and cytochrome $C$ release (Figures $2 \mathbf{A}, \mathbf{B}$ ). Moreover, the exposure of HS to cardiomyocytes also resulted in the cleavage (activation) of caspase 3 (Figures 2C,E,F). The effector caspase 3 is a pro-apoptotic caspase that is activated by mitochondrial cytochrome $\mathrm{C}$ and induces apoptosis in its activated form. Furthermore, we found increased levels of the cleaved product of PARP, indicating the activation of the pro-apoptotic signal cascade (Figure 2D). Cleaved PARP is a by-product of the proapoptotic signal cascade. PARP, normally involved in DNA repair, 

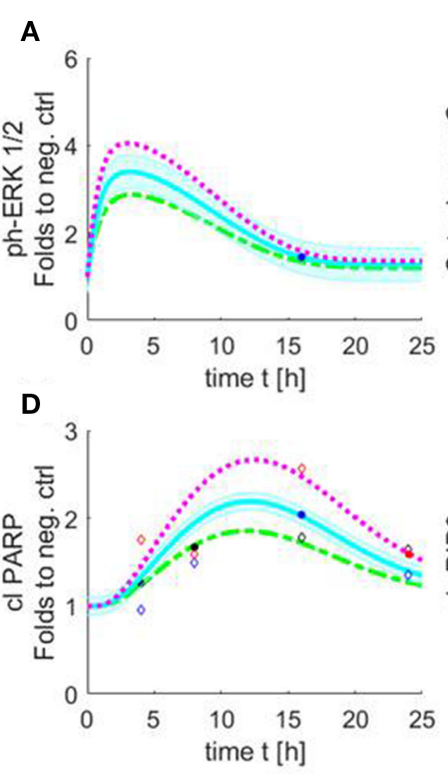

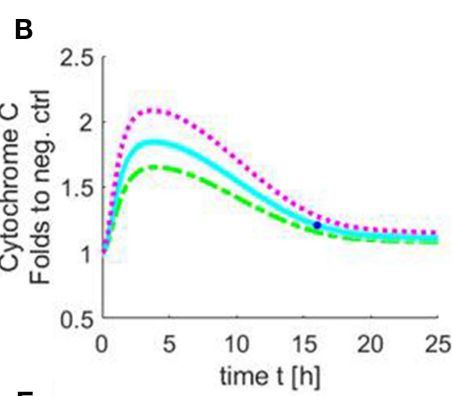

E

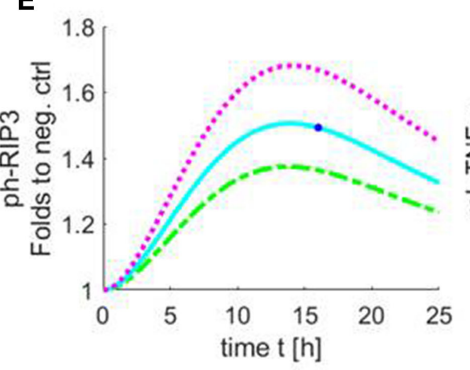

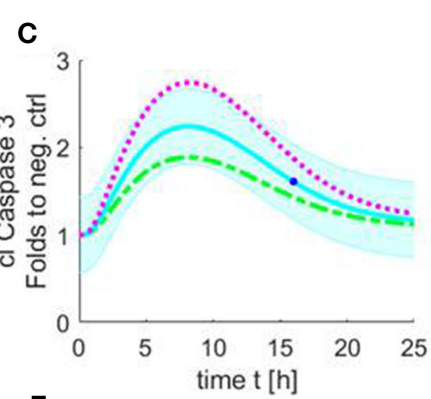

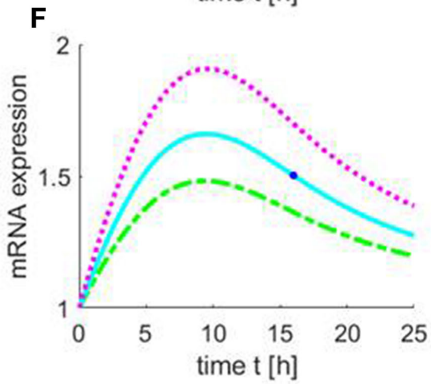

FIGURE 9 | Simulated time course of all involved components of the apoptosis/necroptosis signal pathway induced by HS. Simulated time course of the components involved in intrinsic apoptosis and necroptosis signaling pathways for cardiomyocytes exposed to three different HS concentrations [5 (green), 10 (blue), and 20 (purple) $\mu \mathrm{g} / \mathrm{ml}$ of HS]. The blue background represents the standard error of the measured data. The model was simulated based on relative cleaved PARP values. The filled points in graphs represent data used for training and the unfilled for validation. Simulated time course for relative protein expression of (A) phosphorylation of ERK 1/2, (B) cytochrome C, (C) cleaved caspase 3, (D) cleaved PARP, and (E) phosphorylation of RIP3 is shown. (F) Simulated data of relative TNF- $\alpha$ mRNA expression. HS, heparan sulfate; ph-ERK, phospho-extracellular signal-regulated kinase; PARP, poly-(ADP-ribose) polymerase; RIP, receptor-interacting protein; TNF- $\alpha$, tumor necrosis factor alpha.

is cleaved and subsequently deactivated by the pro-apoptoticcleaved caspase 3 (46). Our results indicate that the induction of the pro-apoptotic cascade by HS may proceed through the intrinsic pathway (Figure 2) (47).

\section{HS Induces Necroptosis in Cardiomyocytes}

Using TUNEL, we could not detect any apoptotic cardiomyocytes after the exposure to HS. Flow cytometry using Annexin V/7AAD staining and determination of cell vitality using PI/Hoechst staining showed that HS (within $16 \mathrm{~h}$ ) causes cell death secondary to necrosis, rather than apoptosis (Figures 3 and 4). Moreover, the simulation showed a peak of caspase 3 activation (Figure 9C) already after $8 \mathrm{~h}$, suggesting that $16 \mathrm{~h}$ of HS exposure might be too long to detect apoptosis in cardiomyocytes, which indeed was not detectable in our in vitro experiments (Figures 3 and 4). Necrosis is known and described as an unprogrammed cell death; however, Degterev et al. described a programmed form of necrosis, named necroptosis (48). TNF- $\alpha$ is a pro-inflammatory cytokine that induces necroptosis by activation of RIP3 and MLKL $(49,50)$. More specifically, TNF- $\alpha$ induces the activation (phosphorylation) of RIP3, which forms a necrosome with MLKL and, hence, induces necroptosis (9). Indeed, the exposure of cardiomyocytes to HSs resulted in an increased, relative TNF- $\alpha$ mRNA expression (Figure 5A), confirming previous data of our group that showed higher TNF- $\alpha$ levels in the supernatant of cardiomyocytes exposed to HS (30). In line with this finding, we also found that RIP3 and MLKL activation was increased in cardiomyocytes exposed to HS for $16 \mathrm{~h}$ (Figures 5B,C). In addition, the simulation results showed the highest relative expression of TNF- $\alpha$
(Figure 9F) and the highest phosphorylation of RIP3 (Figure 9E) between 10 and $16 \mathrm{~h}$, underlying the in vitro detected necroptosis after $16 \mathrm{~h}$ exposure to HS. The phosphorylation of RIP3 is essential and represents a pivotal pathway in the necroptosis induced by TNF- $\alpha$, as phosphorylated RIP3 forms together with phosphorylated MLKL the necrosome, which ultimately induces necroptosis (51). Intracellular ATP levels also play a role in the crosstalk between apoptosis and necrosis, as lower ATP concentrations result in necrosis, while higher ATP concentrations drive apoptosis (52). Notably, we recently showed that the exposure of cardiomyocytes to HS results in lower ATP concentrations in cardiomyocytes (12), possibly secondary to excessive activation of PARP (53).

\section{Simulated Time Course}

As the use of in vitro experiments to investigate pathophysiological processes is complex, time-consuming, and expensive, we aimed to investigate a new and very promising solution to overcome these shortcomings of "classical" experimental techniques using computer-based methods. Our Medical-In silico approach proposes an alternative to running a wide range of experiments with the objective of finding interesting points such as maximum and minimum, which is costly. The optimizer offers a function describing the relationship between the variables under investigation and time from a single training data set. Further experimental data points suggested by the in silico experiments can be used to further develop the model. This facilitates the accumulation of knowledge from experimental data and minimizes the number of needed experiments to be used by the optimizer. The simulated 
results (Figure 9) indicated that the optimization process offered solutions that fitted all the experimental data for all experiments. In addition, some of the experimental data sets were not used in the training process to be used for verification (Figure 9D). The mean deviation of these verification data was $20.49 \%$. Given the low number of training and verification data relative to the system complexity, the verification process is non-conclusive; however, this verification process constitutes an indication to the system learning convergence, as a fully non-trained system has a higher mean SD $(\sim 50 \%)$.

\section{Limitation/Conclusion}

As our investigation is limited to in vitro analyses, further in vivo studies have to confirm the role of HS-induced necroptosis in the pathophysiology of sepsis- and trauma-associated cardiomyopathy. Moreover, the simulated data were limited to a small set of data gained from in vitro experiments. In conclusion, our data showed for the first time that HS induces necroptosis in cardiomyocytes by phosphorylation (activation) of RIP3 and indicate that the used Medical-In silico approach (as a proof-of-concept) is a first step toward simulating the extent of activated components in the pro-apoptotic pathway with only a small data set gained from the in vitro experiments by using machine learning algorithms. Additionally, the simulation indicates that a Medical-In silico

\section{REFERENCES}

1. World Health Organization. Violence and Injuries: The Facts 2014. (2015). Available from: http://www.who.int/violence_injury_prevention/media/news/ 2015/Injury_violence_facts_2014/en/ (accessed March 7, 2018).

2. Cohen J. The immunopathogenesis of sepsis. Nature (2002) 420(6917):885-91. doi:10.1038/nature01326

3. Martin L, Derwall M, Thiemermann C, Schurholz T. [Heart in sepsis: molecular mechanisms, diagnosis and therapy of septic cardiomyopathy]. Anaesthesist (2017) 66(7):479-90. doi:10.1007/s00101-017-0329-x

4. Wilson NM, Wall J, Naganathar V, Brohi K, De'Ath HD. Mechanisms involved in secondary cardiac dysfunction in animal models of trauma and hemorrhagic shock. Shock (2017) 48(4):401-10. doi:10.1097/SHK.0000000000000882

5. Li S, Tao L, Jiao X, Liu H, Cao Y, Lopez B, et al. TNFalpha-initiated oxidative/nitrative stress mediates cardiomyocyte apoptosis in traumatic animals. Apoptosis (2007) 12(10):1795-802. doi:10.1007/s10495-007-0108-2

6. Tao L, Liu HR, Gao F, Qu Y, Christopher TA, Lopez BL, et al. Mechanical traumatic injury without circulatory shock causes cardiomyocyte apoptosis: role of reactive nitrogen and reactive oxygen species. Am J Physiol Heart Circ Physiol (2005) 288(6):H2811-8. doi:10.1152/ajpheart.01252.2004

7. Yao X, Wigginton JG, Maass DL, Ma L, Carlson D, Wolf SE, et al. Estrogenprovided cardiac protection following burn trauma is mediated through a reduction in mitochondria-derived DAMPs. Am J Physiol Heart Circ Physiol (2014) 306(6):H882-94. doi:10.1152/ajpheart.00475.2013

8. Kerr JF, Wyllie AH, Currie AR. Apoptosis: a basic biological phenomenon with wide-ranging implications in tissue kinetics. Br J Cancer (1972) 26(4):239-57. doi:10.1038/bjc.1972.33

9. Linkermann A, Green DR. Necroptosis. N Engl J Med (2014) 370(5):455-65. doi:10.1056/NEJMra1310050

10. Carlson DL, Lightfoot E Jr, Bryant DD, Haudek SB, Maass D, Horton J, et al. Burn plasma mediates cardiac myocyte apoptosis via endotoxin. Am J Physiol Heart Circ Physiol (2002) 282(5):H1907-14. doi:10.1152/ajpheart.00393.2001

11. Goodall KJ, Poon IKH, Phipps S, Hulett MD. Soluble heparan sulfate fragments generated by heparanase trigger the release of pro-inflammatory cytokines through TLR-4. PLoS One (2014) 9(10):e109596. doi:10.1371/journal.pone. 0109596

12. Martin L, Peters C, Schmitz S, Moellmann J, Martincuks A, Heussen N, et al. Soluble heparan sulfate in serum of septic shock patients induces mitochondrial approach, as performed in this study, can help to identify the right time point for further measurements and indeed replaces further time-consuming and cost-intense in vitro experiments.

\section{AUTHOR CONTRIBUTIONS}

Conception and design: EZ, LM, GD, AS, and CT. In vitro experiments and data analyses: EZ, LM, TS, T-PS, AM, GM-N, OK, GM, and PV. Medical in silico experiments and data analyses: EZ, $P V, J Z, G D, A S, L M, A H$, and GA. EZ wrote the manuscript. Correction of the manuscript: EZ, PV , LM , CT, GM , GD , T -PS, and AS. All the authors reviewed and finally approved the manuscript.

\section{ACKNOWLEDGMENTS}

This work was supported by an intramural grant to LM (START 46/16) and EZ (START 113/17). LM has received a grant by the Deutsche Forschungsgemeinschaft (DFG, MA 7082/1-1). We thank Dr Claycomb and his coworkers for providing the HL-1 cells and a detailed documentation. The Immunohistochemistry and Confocal Microscopy Unit, a core facility of the Interdisciplinary Center for Clinical Research (IZKF) Aachen, within the Faculty of Medicine at the RWTH Aachen University, supported this work.

dysfunction in murine cardiomyocytes. Shock (2015) 44(6):569-77. doi:10.1097/SHK.0000000000000462

13. Maeda N. Proteoglycans and neuronal migration in the cerebral cortex during development and disease. Front Neurosci (2015) 9:98. doi:10.3389/fnins.2015. 00098

14. Martin L, Koczera P, Zechendorf E, Schuerholz T. The endothelial glycocalyx: new diagnostic and therapeutic approaches in sepsis. Biomed Res Int (2016) 2016:3758278. doi:10.1155/2016/3758278

15. Sarrazin S, Lamanna WC, Esko JD. Heparan sulfate proteoglycans. Cold Spring Harb Perspect Biol (2011) 3(7):a004952. doi:10.1101/cshperspect.a004952

16. Gilbert D, Fuss H, Gu X, Orton R, Robinson S, Vyshemirsky V, et al. Computational methodologies for modelling, analysis and simulation of signalling networks. Brief Bioinform (2006) 7(4):339-53. doi:10.1093/bib/ bbl043

17. Habibi I, Emamian ES, Abdi A. Quantitative analysis of intracellular communication and signaling errors in signaling networks. BMC Syst Biol (2014) 8(1):89. doi:10.1186/s12918-014-0089-z

18. Kitano H. Computational systems biology. Nature (2002) 420(6912):206-10. doi:10.1038/nature01254

19. Banga JR. Optimization in computational systems biology. BMC Syst Biol (2008) 2(1):47. doi:10.1186/1752-0509-2-47

20. Moles CG, Mendes P, Banga JR. Parameter estimation in biochemical pathways: a comparison of global optimization methods. Genome Res (2003) 13(11):2467-74. doi:10.1101/gr.1262503

21. Mendes P, Kell D. Non-linear optimization of biochemical pathways: applications to metabolic engineering and parameter estimation. Bioinformatics (1998) 14(10):869-83. doi:10.1093/bioinformatics/14.10.869

22. Hansen N. The CMA Evolution Strategy: A Tutorial. (2010). Available from: https://arxiv.org/pdf/1604.00772v1.pdf (accessed March 7, 2018).

23. Heiner M, Gilbert D, Donaldson R. Petri nets for systems and synthetic biology. International School on Formal Methods for the Design of Computer, Communication and Software Systems. Berlin, Heidelberg: Springer (2008). p. 215-64.

24. Breitling R, Gilbert D, Heiner M, Orton R. A structured approach for the engineering of biochemical network models, illustrated for signalling pathways. Brief Bioinform (2008) 9(5):404-21. doi:10.1093/bib/bbn026

25. Heiner M, Koch I, Will J. Model validation of biological pathways using Petri nets - demonstrated for apoptosis. Biosystems (2004) 75(1-3):15-28. doi:10.1016/j.biosystems.2004.03.003 
26. Hardy S, Robillard PN. Modeling and simulation of molecular biology systems using petri nets: modeling goals of various approaches. J Bioinform Comput Biol (2004) 2(04):619-37. doi:10.1142/S0219720004000764

27. Blätke MA, Heiner M, Marwan W. Biomodel engineering with petri nets. Algebraic and Discrete Mathematical Methods for Modern Biology. Amsterdam: Elsevier (2015). p. 141-92.

28. Wolkenhauer O, Ullah M, Kolch W, Cho K-H. Modeling and simulation of intracellular dynamics: choosing an appropriate framework. IEEE Trans Nanobioscience (2004) 3(3):200-7. doi:10.1109/TNB.2004.833694

29. Claycomb WC, Lanson NA, Stallworth BS, Egeland DB, Delcarpio JB, Bahinski A, et al. HL-1 cells: A cardiac muscle cell line that contracts and retains phenotypic characteristics of the adult cardiomyocyte. Proc Natl Acad Sci U S A (1998) 95(6):2979-84. doi:10.1073/pnas.95.6.2979

30. Martin L, Schmitz S, De Santis R, Doemming S, Haase H, Hoeger J, et al. Peptide 19-2.5 inhibits heparan sulfate-triggered inflammation in murine cardiomyocytes stimulated with human sepsis serum. PLoS One (2015) 10(5):e0127584. doi:10.1371/journal.pone.0143583

31. Martin L, Horst K, Chiazza F, Oggero S, Collino M, Brandenburg K, et al. The synthetic antimicrobial peptide 19-2.5 attenuates septic cardiomyopathy and prevents down-regulation of SERCA2 in polymicrobial sepsis. Sci Rep (2016) 6:37277. doi:10.1038/srep37277

32. Gavrieli Y, Sherman Y, Ben-Sasson SA. Identification of programmed cell death in situ via specific labeling of nuclear DNA fragmentation. J Cell Biol (1992) 119(3):493-501. doi:10.1083/jcb.119.3.493

33. David RENÉ, Alla H. Discrete, continuous, and hybrid Petri nets. IEEE Control Systems (2008) 28(3):81-4. doi:10.1109/MCS.2008.920445

34. Shampine LF, Reichelt MW. The matlab ode suite. SIAM J Sci Comput (1997) 18(1):1-22. doi:10.1137/S1064827594276424

35. Hansen N. The CMA evolution strategy: a comparing review. Towards a New Evolutionary Computation. Berlin, Heidelberg: Springer (2006). p. 75-102.

36. Hansen N, Muller SD, Koumoutsakos P. Reducing the time complexity of the derandomized evolution strategy with covariance matrix adaptation (CMAES). Evol Comput (2003) 11(1):1-18. doi:10.1162/106365603321828970

37. Klipp E, Liebermeister W, Wierling C, Kowald A, Herwig R. Systems Biology: A Textbook. New Jersey: John Wiley \& Sons (2016).

38. Horn F, Jackson R. General mass action kinetics. Arch Rational Mech Anal (1972) 47(2):81-116. doi:10.1007/BF00251225

39. van Riel NA, Sontag ED. Parameter estimation in models combining signal transduction and metabolic pathways: the dependent input approach. Syst Biol (Stevenage) (2006) 153(4):263-74. doi:10.1049/ip-syb: 20050076

40. Koch I, Junker BH, Heiner M. Application of Petri net theory for modelling and validation of the sucrose breakdown pathway in the potato tuber. Bioinformatics (2004) 21(7):1219-26. doi:10.1093/bioinformatics/bti145

41. Formanowicz D, Radom M, Zawierucha P, Formanowicz P. Petri net-based approach to modeling and analysis of selected aspects of the molecular regulation of angiogenesis. PLoS One (2017) 12(3):e0173020. doi:10.1371/journal. pone. 0173020
42. Sharma SK, Naidu G. The role of danger-associated molecular patterns (DAMPs) in trauma and infections. J Thorac Dis (2016) 8(7):1406-9. doi:10.21037/jtd.2016.05.22

43. Elmore S. Apoptosis: a review of programmed cell death. Toxicol Pathol (2007) 35(4):495-516. doi:10.1080/01926230701320337

44. Cagnol S, Chambard JC. ERK and cell death: mechanisms of ERK-induced cell death - apoptosis, autophagy and senescence. FEBS J (2010) 277(1):2-21. doi:10.1111/j.1742-4658.2009.07366.x

45. Seo HJ, Choi SJ, Lee JH. Paraquat induces apoptosis through cytochrome C release and ERK activation. Biomol Ther (2014) 22(6):503-9. doi:10.4062/ biomolther.2014.115

46. Pieper AA, Verma A, Zhang J, Snyder SH. Poly (ADP-ribose) polymerase, nitric oxide and cell death. Trends Pharmacol Sci (1999) 20(4):171-81. doi:10.1016/ S0165-6147(99)01292-4

47. Li P, Nijhawan D, Budihardjo I, Srinivasula SM, Ahmad M, Alnemri ES, et al. Cytochrome $c$ and dATP-dependent formation of Apaf-1/caspase- 9 complex initiates an apoptotic protease cascade. Cell (1997) 91(4):479-89. doi:10.1016/ S0092-8674(00)80434- 1

48. Degterev A, Huang Z, Boyce M, Li Y, Jagtap P, Mizushima N, et al. Chemical inhibitor of nonapoptotic cell death with therapeutic potential for ischemic brain injury. Nat Chem Biol (2005) 1(2):112-9. doi:10.1038/nchembio711

49. Linkermann A, Stockwell BR, Krautwald S, Anders HJ. Regulated cell death and inflammation: an auto-amplification loop causes organ failure. Nat Rev Immunol (2014) 14(11):759-67. doi:10.1038/nri3743

50. Jaco I, Annibaldi A, Lalaoui N, Wilson R, Tenev T, Laurien L, et al. MK2 phosphorylates RIPK1 to prevent TNF-induced cell death. Mol Cell (2017) 66(5):698.e-710.e. doi:10.1016/j.molcel.2017.05.003

51. Moriwaki K, Chan FK. RIP3: a molecular switch for necrosis and inflammation. Genes Dev (2013) 27(15):1640-9. doi:10.1101/gad.223321.113

52. Nikoletopoulou V, Markaki M, Palikaras K, Tavernarakis N. Crosstalk between apoptosis, necrosis and autophagy. Biochim Biophys Acta (2013) 1833(12):3448-59. doi:10.1016/j.bbamcr.2013.06.001

53. Thiemermann C, Bowes J, Myint FP, Vane JR. Inhibition of the activity of poly(ADP ribose) synthetase reduces ischemia-reperfusion injury in the heart and skeletal muscle. Proc Natl Acad Sci U S A (1997) 94(2):679-83. doi:10.1073/ pnas.94.2.679

Conflict of Interest Statement: The authors declare that the research was conducted in the absence of any commercial or financial relationships that could be construed as a potential conflict of interest.

Copyright (c) 2018 Zechendorf, Vaßen, Zhang, Hallawa, Martincuks, Krenkel, MüllerNewen, Schuerholz, Simon, Marx, Ascheid, Schmeink, Dartmann, Thiemermann and Martin. This is an open-access article distributed under the terms of the Creative Commons Attribution License (CC BY). The use, distribution or reproduction in other forums is permitted, provided the original author(s) and the copyright owner are credited and that the original publication in this journal is cited, in accordance with accepted academic practice. No use, distribution or reproduction is permitted which does not comply with these terms. 